\title{
ON-LINE CALIBRATION AND DIGITAL CORRECTION OF MULTI-BIT SIGMA-DELTA MODULATORS
}

\author{
A. Fornasari, F. Borghetti, P. Malcovati and F. Maloberti \\ Dept. of Electrical Engineering, University of Pavia, \\ Via Ferrata 1, 27100 Pavia, Italy \\ E-mail: \{andrea.fornasari, fausto.borghetti, piero.malcovati,franco.maloberti\}@unipv.it
}

\begin{abstract}
This paper presents a new method for the on-line calibration of the elements of the digital-to-analog converter (DAC) used in the feedback loop of multi-bit sigma-delta $(\Sigma \Delta)$ modulators. The proposed method, with an extremely limited amount of additional circuitry, allows the mismatches among the elements of the DAC to be accurately measured and corrected in the digital domain, thus improving the linearity performance of the modulator. A switched-capacitor (SC) 4-bit $\Sigma \Delta$ modulator has been implemented to validate the proposed solution. The experimental results confirm that this method allows us to achieve an improvement in the spurious-free dynamic range as large as $26 \mathrm{~dB}$.
\end{abstract}

\section{INTRODUCTION}

Sigma-delta $(\Sigma \Delta)$ modulators can be realized using single-bit or multi-bit digital-to-analog converters (DACs) in the feedback loop. When a single-bit DAC is used, the modulator is inherently linear and therefore the DAC element matching is not important at all. The use of a multibit DAC in the feedback loop allows us to increase the modulator resolution without requiring to increase the oversampling ratio or the order of modulator. In this case, however, the linearity of the whole $\Sigma \Delta$ modulator is limited by the linearity of the DAC used. Therefore, for multi-bit $\Sigma \Delta$ modulators the correction of the mismatches among the elements of the DAC to achieve high linearity becomes one of the major design issue.

A widely used solution for this problem is the dynamic element matching (DEM) technique $[1,2]$. With this method the DAC elements used for each conversion are selected randomly. The result is that the mismatches among the DAC elements, instead of degrading the modulator linearity, lead to a wide-band noise. In some implementations this mismatch noise is high-pass filtered, thus diminishing the contribution that falls in the baseband. Since the DEM techniques are not actually correcting the DAC element mismatches, but rely on the transformation of the mismatch effects into noise, when under particular conditions this result is only partially achieved, distortion tones appear, causing a degradation of the spurious-free dynamic range.

This paper describes a technique for actually measuring on-line the mismatches among the DAC elements. The obtained errors are stored in a memory and fully corrected in the digital domain. The experimental measurements on a 4-bit second order $\Sigma \Delta$ modulator validate this method. The proposed approach reduces the harmonic distortion caused by the mismatch among the DAC elements by more than $26 \mathrm{~dB}$. The area penalty for the implementation of this method is very limited and comparable with the area required for implementing a DEM technique for a 4-bit DAC.

\section{Proposed TeChNiQue}

The on-line calibration of the unity elements of a DAC requires the use of an extra element in the DAC and a reference element. While the circuit operates regularly one element of the DAC is selected and compared with the reference element. At the end, another element is selected and measured until all the elements are calibrated. The approach requires that the measurement process does not interfere with the normal operation of the $\Sigma \Delta$ modulator. Since a $\Sigma \Delta$ modulator is based on oversampling, there is a wide range of the spectrum between the edge of the baseband and the Nyquist frequency which is of no interest and could be exploited for measuring the mismatch. However, this frequency range is affected by significant quantization noise.

The basic idea behind the proposed technique is to create a noise-free frequency region outside the band of interest and use this part of the spectrum, through a suitable modulation, for measuring the mismatch. In the proposed implementation we selected a region around half of the sampling frequency $\left(f_{S} / 2\right)$. That means that the noise transfer function $(N T F)$ of the $\Sigma \Delta$ modulator has to include a notch at $f_{S} / 2$. An important point for the circuit realization is the minimization of the hardware penalty. Typically, in order to add a notch in the NTF an additional operational amplifier is required in the modulator. In the proposed implementation, we achieved this results for a second order modulator without using any additional active device, thus making the hardware penalty negligible. 


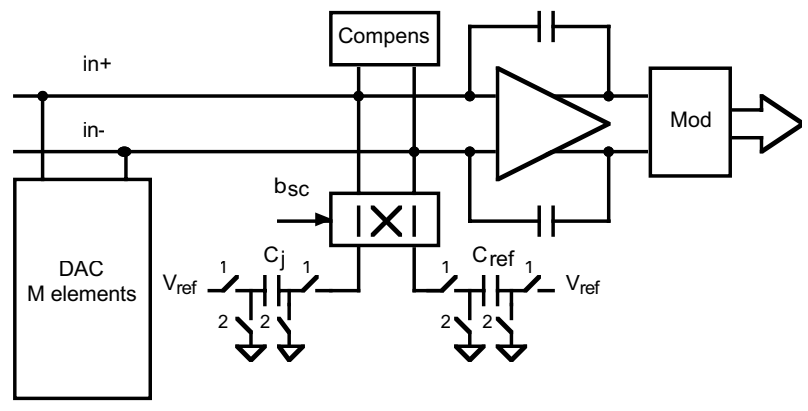

Figure 1: Modified $\Sigma \Delta$ modulator for capacitor mismatch measurement

Fig. 1 illustrates the proposed method used to measure the mismatch in a capacitor-based DAC. The capacitive array consists of $M+1$ unity capacitors. While $M$ capacitors perform the normal digital-to-analog conversion, one of them $\left(C_{j}\right)$ is under calibration. The switched-capacitor (SC) structure including $C_{j}$ injects in the feedback capacitor of the integrator a charge equal to $C_{j} V_{r e f}$ every clock cycle, while the SC branch including the reference element $\left(C_{r e f}\right)$ injects a charge $C_{r e f} V_{r e f}$. If $C_{j}=C_{r e f}$, the injected charges represent a common mode signal that does not affect the differential output of the integrator. In fact, a suitable compensation network cancels this common-mode term. On the other hand, a mismatch between $C_{j}$ and $C_{r e f}$ determines a differential contribution. A mixer, driven by a binary signal $b_{s c}$, controls the injection of the mismatch signal. In this case, the mixer modulates the mismatch signal at $f_{S} / 2$. Since the region of the spectrum around $f_{S} / 2$ is free from the quantization noise, it is possible to extract the mismatch information from the output bitstream, without affecting the input signal. This is achieved by placing a second identical mixer in the digital domain at the output of the $\Sigma \Delta$ modulator, thus down-converting at DC the mismatch error.

The value of the error is then extracted with a simple digital low-pass filter. The desired accuracy in the error measurement can be achieved by reducing the bandwidth of the filter used. In practical cases, a digital integrate-anddump circuit with a long integration period is sufficient. The calibration operation, of course, has to be repeated (if wished periodically in time) for all the DAC elements. The information on the mismatch between each DAC element and the reference element is stored in a look-up table. To calibrate the $\Sigma \Delta$ modulator, canceling the effect of the DAC element mismatch, the stored error values have to be subtracted in the digital domain from the modulator output bitstream. This could be performed directly at the output of the modulator before the decimation filter. However, in order to achieve the desired resolution, the mismatch measurement must be very accurate, thus strongly increasing the word-length of bitstream after the subtraction and hence the complexity of the decimation filter itself. Instead, it is more effective to filter the two signals $(\Sigma \Delta$

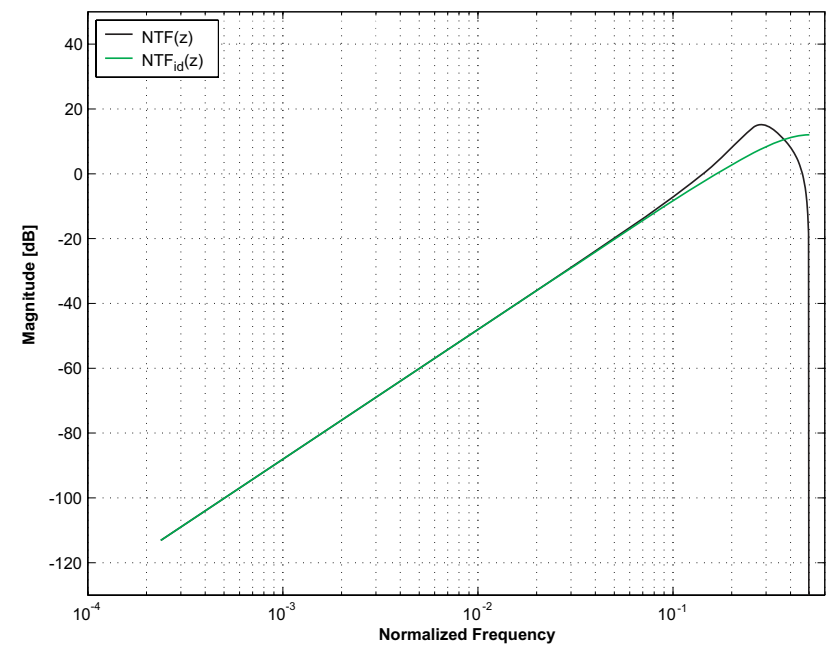

Figure 2: NTF of a traditional 2nd order modulator and of the proposed one

modulator output bitstream and mismatch error) independently, and to combine the results after decimation.

\section{A/D CONVERTER IMPLEMENTATION}

The implementation of the proposed technique requires to design a $\Sigma \Delta$ modulator including a term $(z+1)$ in the numerator of the $N T F$, in order to introduce the additional transmission zero at $f_{S} / 2$. Moreover, the noise shaping at low frequency must remain unmodified. Finally, the signal transfer function must be flat in the signal band. All, the above features must be obtained without increasing the number of operational amplifiers used. The following signal transfer function $(S T F)$ and noise transfer function $(N T F)$ fulfill the above mentioned constraints for a second order $\Sigma \Delta$ modulator:

$$
\begin{aligned}
& \operatorname{STF}(z)=\frac{3.5 z-1.5}{z^{3}+0.4 z^{2}+0.5 z+0.1} \\
& \operatorname{NTF}(z)=\frac{(z-1)^{2}(z+1)}{z^{3}+0.4 z^{2}+0.5 z+0.1}
\end{aligned}
$$

In addition to a notch at $f_{S} / 2$, these $\Sigma \Delta$ modulator transfer functions shows, at low frequency $(z=1)$, the same noise shaping of a conventional second order modulator:

$$
N T F_{i d}(z)=(z-1)^{2}
$$

as illustrated in Fig. 2.

The $\Sigma \Delta$ modulator architecture that implements the $S T F$ and the NTF of (1) and (2) is shown in Fig. 3. In this modulator the second integrator has been replaced with a biquadratic cell, in order to realized the additional zero in the NTF. Even if in literature [3, 4] several ways to implement a biquadratic transfer function using only one operation amplifier are available, we chose to use an N-path filter, as shown in Fig. 4. Indeed, thanks to the position of 


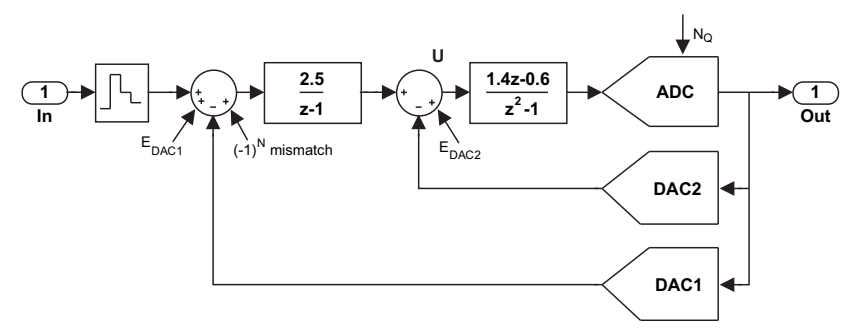

Figure 3: $\Sigma \Delta$ modulator architecture implementing the desired transfer functions

this block in the modulator loop, any non-ideality associated with it benefits of a first order shaping, thus making the path-mismatch, which is the most relevant drawback of this kind of filter [4], negligible. For the same reason, only the errors caused by the first DAC (DAC1) are important: errors introduced by the second DAC (DAC2), in fact, become irrelevant because of the first order shaping. Therefore, we chose to use the proposed method only for DAC1.

During the calibration phase the data converter output is

$$
\begin{aligned}
\text { Out }= & \operatorname{In} \cdot \operatorname{STF}(z)+E_{D A C 1} \cdot \operatorname{STF}(z)+E_{D A C 2} \cdot \operatorname{ETF}(z)+\ldots \\
& \ldots+N_{Q} \cdot \operatorname{NTF}(z)+M i{ }^{*} \cdot \operatorname{STF}(z)
\end{aligned}
$$

where $E_{D A C 1}$ and $E_{D A C 2}$ are the errors introduced by DAC1 and DAC2, respectively, ETF is the transfer function from node $U$ to the output, $N_{Q}$ is the quantization noise and $\mathrm{Mis}^{*}$ is the DAC mismatch modulated by $b_{s c}$. Since the input signal is band-limited, the output spectrum contains two components: one due to $\operatorname{In} \cdot \operatorname{STF}(1)+E_{D A C 1} \cdot \operatorname{STF}(1)$ in the

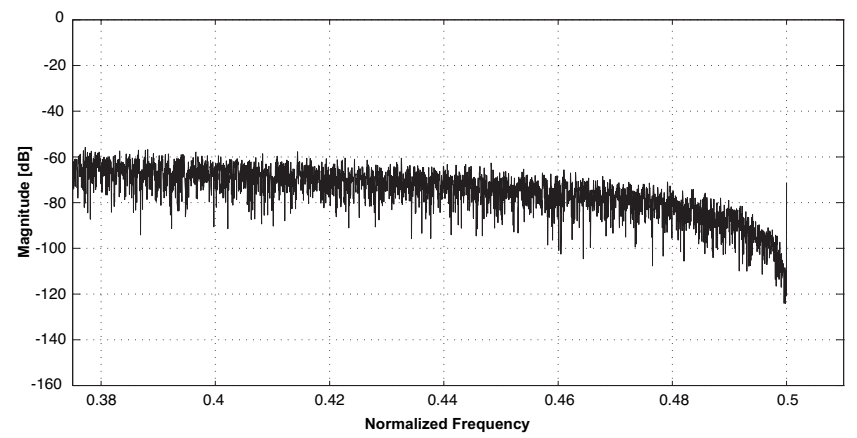

Figure 5: Calibration tone located at half of the sampling frequency

baseband and one caused by $\operatorname{Mis}^{*} \cdot \operatorname{STF}(-1)$ at high frequency, as shown in Fig. 5. As already discussed, the mismatch can be extracted multiplying the output bitstream by \pm 1 .

We achieve mismatch correction in the digital domain by subtracting from the output the estimated mismatch $<E_{D A C 1}>$ divided by $\operatorname{STF}(-1)$. Assuming that the estimation differs from the real error by $Q_{<E D A C 1>}$ we have

$$
\begin{aligned}
& \operatorname{In} \cdot \operatorname{STF}(z)+E_{D A C 1} \cdot \operatorname{STF}(z)-\left\langle E_{D A C 1}\right\rangle \cdot \operatorname{STF}(z)= \\
& =\operatorname{In} \cdot \operatorname{STF}(z)+Q_{\left\langle E_{D A C 1}\right\rangle} \cdot \operatorname{STF}(z)
\end{aligned}
$$

Therefore, if we want to make the residual error smaller than the quantization contribution it is necessary to use a very accurate estimation of $E_{D A C 1}$. Fig. 6 shows the block diagram of the digital correction hardware.

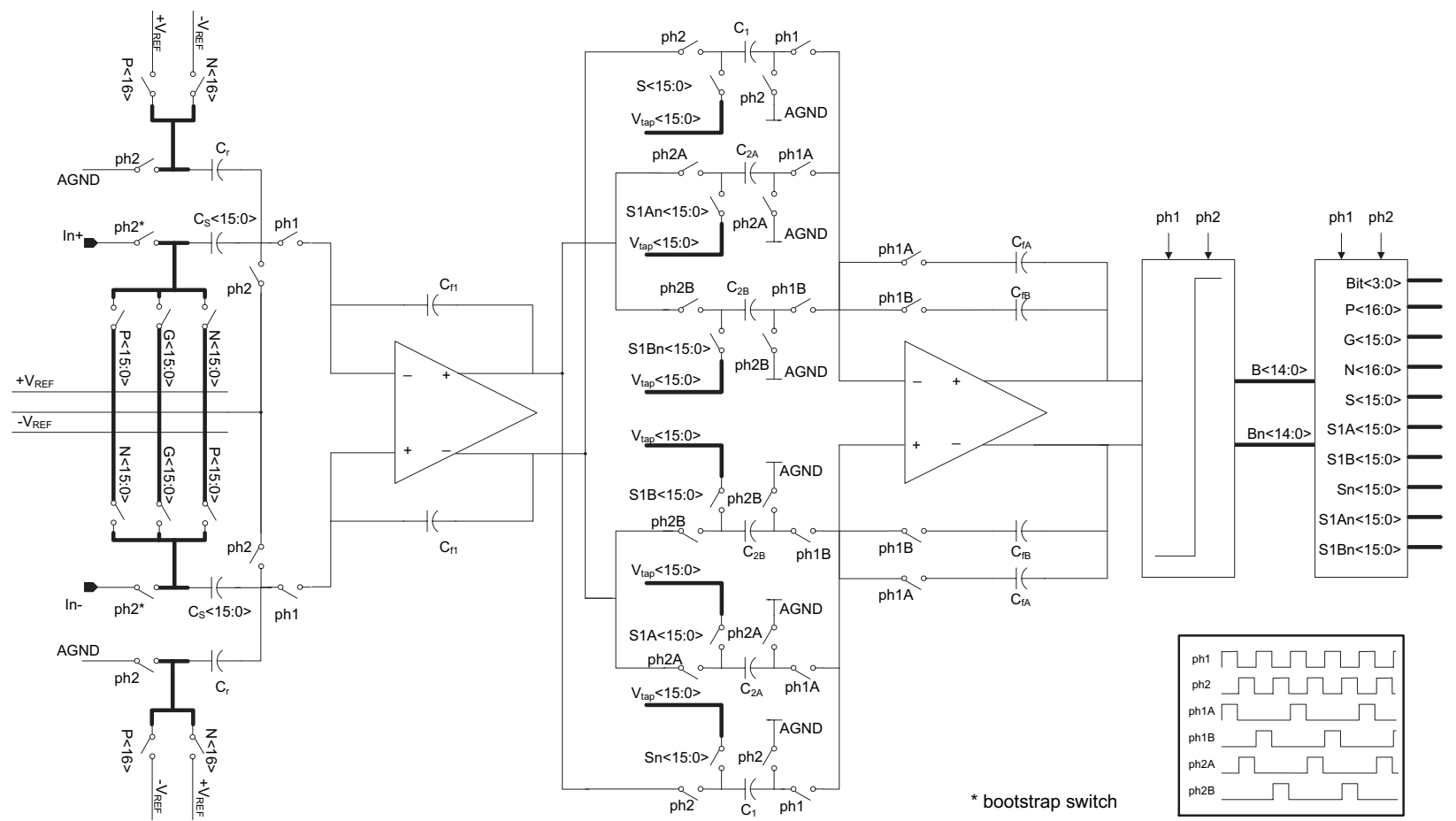

Figure 4: Schematic of the proposed $\Sigma \Delta$ modulator 


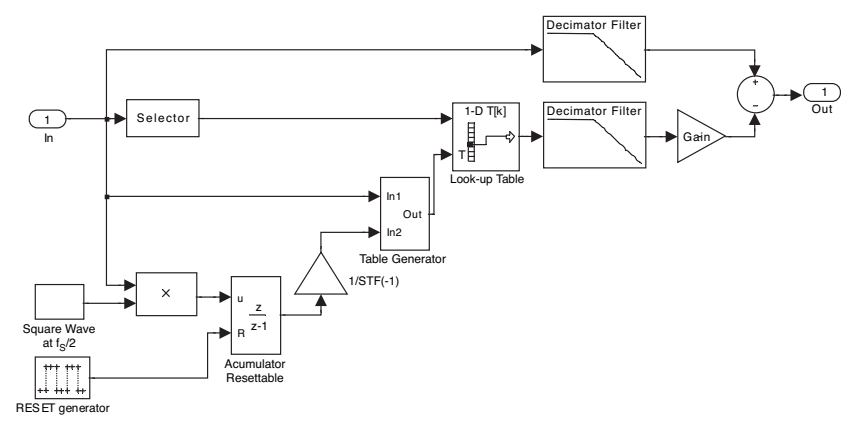

Figure 6: Principle scheme of the proposed digital correction circuit

The error word-length in the look-up table depends on the expected mismatch between elements. In order to optimize the look-up table dimension it is useful to have, for the technology used, an estimation of the expected accuracy. Considering that the presented implementation uses 4-bit DACs and assuming a mismatch of $0.2 \%$, only 4 bits words are required for achieving an accuracy of 14 bit. Therefore, the size of the used memory is pretty small. The expected matching also determines the integration time of the digital integrator with reset. Simulation results show that using $2^{17}$ clock periods is more than adequate for the implemented system. Therefore, we complete an entire calibration cycle within $2^{21}$ clock periods.

\section{EXPERIMENTAL RESULTS}

The proposed $\Sigma \Delta$ modulator has been implemented in a $0.35 \mu \mathrm{m}$ CMOS technology. A microphotograph of the $6 \mathrm{~mm}^{2}$ chip is shown in Fig. 7. The power spectral density of the modulator output before and after calibration is shown in Fig. 8. An improvement of more than $30 \mathrm{~dB}$ on the third harmonic has been obtained, leading to a spurious-free dynamic range $(S F D R)$ improvement as large as $26 \mathrm{~dB}$. The proposed technique has no effect on the even harmonics because they are not introduced by the DAC (completely fully differential) but by the signal source. The additional hardware in the analog section required for implementing the proposed technique is extremely limited: only two extra capacitors are needed. The most important features of the proposed modulator are summarized in Table 1.

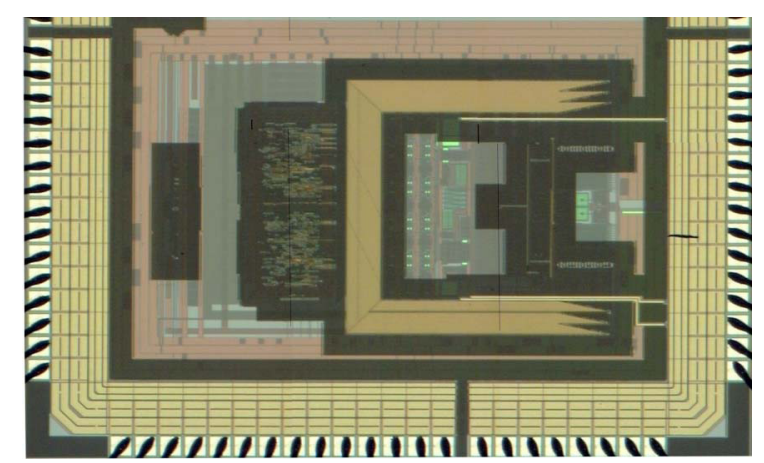

Figure 7: Die photo of the proposed second-order $\Sigma \Delta$ modulator

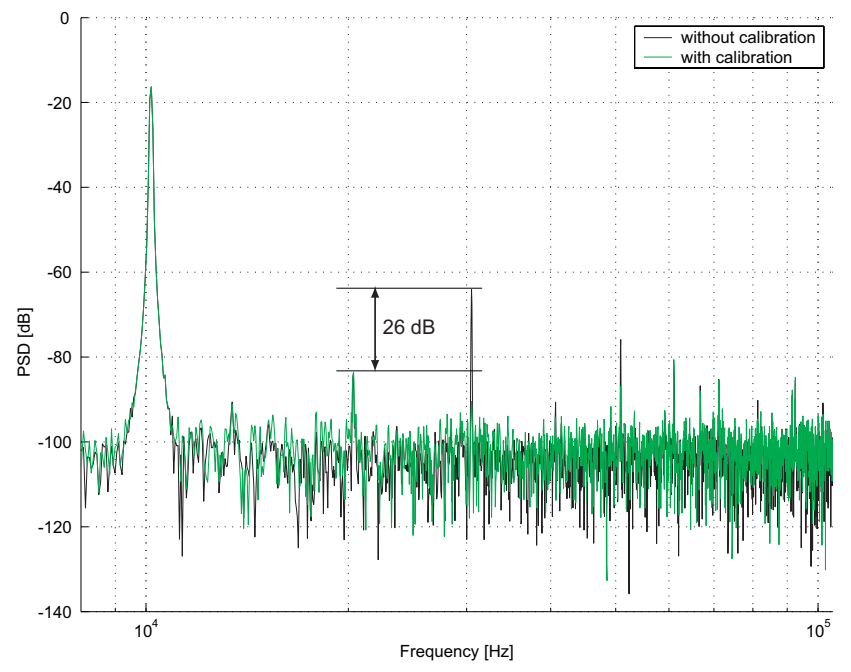

Figure 8: Measured $\Sigma \Delta$ modulator output spectrum

\begin{tabular}{|l|c|}
\hline \multicolumn{1}{|c|}{ Parameter } & Value \\
\hline \hline Area (including pads) & $6 \mathrm{~mm}^{2}$ \\
\hline Sampling frequency & $2.3 \mathrm{MHz}$ \\
\hline Signal bandwidth & $18 \mathrm{kHz}$ \\
\hline SNDR (before and after calibration) & $58.8 \mathrm{~dB}(\mathbf{6 6 . 1} \mathbf{~ d B})$ \\
\hline SFDR (before and after calibration) & $61 \mathrm{~dB}(\mathbf{8 7} \mathbf{~ d B})$ \\
\hline
\end{tabular}

Table 1: Performances of the $\Sigma \Delta$ modulator (input signal at $1 \mathrm{kHz}$ )

\section{CONCLUSIONS}

A method for on-line calibrating the DAC used in multibit sigma-delta modulators has been proposed. The method benefits from an additional transmission zero, located at $f_{S} / 2$, in the modulator noise transfer function. This creates a "clean" area in the output spectrum where it is possible to allocate a tone whose amplitude is proportional to the DAC elements mismatch. A simple digital processing allows us to measure the mismatches and to store them in a digital memory. Measurements on a second-order 4-bit $\Sigma \Delta$ modulator validate the proposed technique, which allows an SFDR improvement by more than $26 \mathrm{~dB}$ to be achieved.

\section{REFERENCES}

[1] I. Galton, "Noise-shaping D/A converters for $\Sigma \Delta$ modulation", Proc. of ISCAS '96, pp. 441-444, May 1996.

[2] Zhimin Li and T.S. Fiez, "Dynamic element matching in low oversampling delta sigma ADCs", Proc. of ISCAS '02, vol. 4, pp. 683-686, May 2002.

[3] P. V. Ananda Mohan, V. Ramachandran, M. N. Swamy, "Parasitic-compensated single amplifier SC biquad equivalent to Fleischer Laker SC biquad", IEEE Trans. on Circuits and Systems, vol. 33, pp. 458-460, Apr. 1986.

[4] G. Palmisano, F. V. G. Espinosa, F. Montecchi, "Performance comparisons of pseudo-N-path SC cells in filters with real operational amplifiers", Proc. of ISCAS '89, pp. 1467-1470, May 1989. 